\title{
A Sensor Scheduling Model of Midcourse Object Tracking Based Signal-to-noise Ratio Optimization
}

\author{
Yuanyuan Yang ${ }^{1,}$, , Weidong Sheng ${ }^{2, \mathrm{~b}}$, Wei $\mathrm{An}^{3, \mathrm{c}}$ \\ ${ }^{1,2,3}$ College of Electronic Science and Engineering, National University of Defense Technology, \\ Changsha, Hunan, P.R. China, 410073 \\ aemail: 352906956@qq.com, bemail: Shengweidong1111@sohu.com, \\ cemail:2516941097@qq.com
}

Keywords: Signal-to-noise optimization; Multi-sensor management; Object continue tracking;

\begin{abstract}
This paper proposes a sensor dynamic scheduling algorithm of midcourse target tracking. This algorithm increases SNR constraints to optimization function which treats Geometric Dilution of Precision (GDOP) as the scheduling criterion. Firstly, by analyzing the different process technologies of midcourse target tracking handoff, this paper proposes a dual-stage of technical idea including recapture confirmation and stable tracking. Secondly, at the recapture confirmation stage, for the purpose of increasing the target recapture probability, this paper verifies that it's necessary and reasonable to increase the target signal to noise ratio as a key constraint in considering the basic factors of the camera detection performance, observed background, stray light and so on. In stable tracking phase, this paper adopts a simplified geometric model which can get higher tracking accuracy to schedule the sensor. Next, The performance of the proposed model are analyzed by using the real particle swarm optimization algorithm that reduces the dimension of the particles and updates the position vector. The simulation results show that the model is more effective.
\end{abstract}

\section{Introduction}

Space Tracking and Surveillance System (STSS) can effectively compensate for Space-Based Infrared System (SBIRS) and ground early warning radar observations gap of the midcourse target, so it is an important means to the middle ballistic target detection [1]. In the system, sensor optimal scheduling is one of the most important and difficult problems to be solved. Generally sensor optimal scheduling is calling the appropriate camera staring in a reasonable time to point to the appropriate airspace achieving the goal of cooperative detection, so that the system task will be completed in the most efficient way. Its essence is a nonlinear optimization problem which establishes an easily quantifiable objective function according to certain criteria, and then we plus sensor resource constraints to optimized the objective function so as to achieve efficient allocation of sensor system [2] .

Sensor scheduling focuses on two aspects: the first is to establish a sensor optimization scheduling model that can reflect the characteristics of the application, the second is to seek suitable sensor scheduling model solution. In the aspect of sensor scheduling model, Wang Bo proposed a sensor scheduling method in the middle of target that used the target tracking accuracy as the optimization goal[3][4], its target tracking accuracy and timeliness all have good performance, but its shortcomings were the lack of consideration of reacquisition process, and the reacquisition failure significantly increased the risk of missing the target handoff. On the basis of this, the paper not only considers the target tracking accuracy, but also focuses on the influence of target reacquisition performance constraints, this paper establishes a midcourse sensor scheduling model based on signal to noise ratio optimization to accommodate target reacquisition performance and tracking accuracy. On solving the model, we use the the real particle swarm optimization (RPSO, Real-number Particle Swarm Optimization) method which reduces the dimension of particles and updates particles location to reduce the amount of computation. The simulation results show that the effectiveness of this method. 


\section{Midcourse Sensor Scheduling Constraints Condition Analysis}

From the standpoint of optimal scheduling, we have to consider the both side of target acquisition and the target tracking accuracy.

\section{The Constraints Condition Analysis In Target Acquisition Stage}

Target reacquisition refers to the process which recaptures the target successfully during the tracking sensor handoff or between the capture sensor and the tracking sensor, it is a process including target space interception, time capture and energy interception all combined factors. The main objective of this article is to consider the impact of energy interception. It's influenced by target energy radiation intensity, background characteristics, load detection performance, the target observation angle and detection distance, sun / moon stray light and other factors. This paper uses the target SNR to measure the impact of target energy interception resulting in establishing a optimization function based on target SNR recapture.

(1) Signal

Figure 1 shows the spaceborne infrared detection system, the signal transmission chain from the radiation source to the voltage / electric current [5].

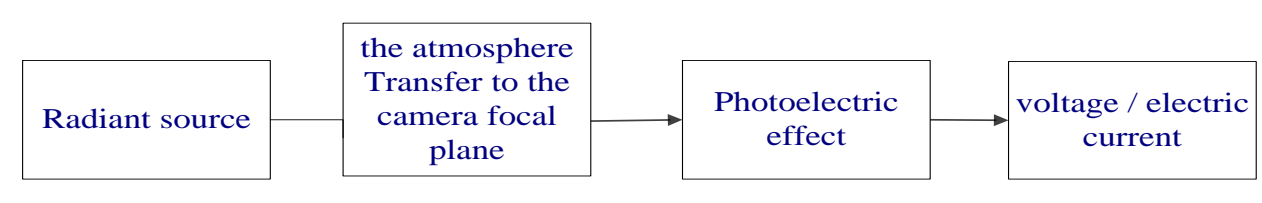

Figure 1. the signal transmission chain in infrared detection system

Since the target is far away from the satellite, it images in the sensor focal plane belonging to "point" target. Under the situation of pixel size, focal length of the optical system is 1 point, the number of electrons target generated in the sensor focal plane is,

$$
N=\frac{A_{0} \tau_{\text {int }}}{h c} \int \frac{I}{R^{2}} \mathrm{o} \lambda \eta \mathrm{o} \tau d \lambda
$$

In the type(1): Ao is optical system entrance pupil area, $\tau_{\text {int }}$ is the integration time, $h$ is Planck's constant, $c$ is the speed of light, $I$ is target spectral irradiance, $R$ is the distance, $\eta$ is quantum efficiency, $\tau$ is the transmission system.

\section{(2) Noise}

Spaceborne infrared staring detection system noise includes background noise, stray light, thermal noise, readout noise.

\section{(a) Background Noise}

The background noise is produced by the progress that background environment observed by the detector shot into the detector. In STSS system, depending on the scheduling result of the sensor there are the following three background for the target detecting, shown in Figure 2.

In the Figure, the satellite height of the orbit is $H$, the Earth's radius is $R$, the dark gray area is the clouds, set its height as $h l$, light gray area is the Earth's outer atmosphere, set its height as $h 2$, tracking sensor points set $P t$, under the stars coordinates pitch angle is $e$, its field of view angle $\delta$, that we can calculate the tracking range of the sensor view using the height $h$ of sight tangent line to represent. Assume that the target missile T appears in the staring camera's field of vision, you can get the type of background according to their line of sight tangent height $h$ : 


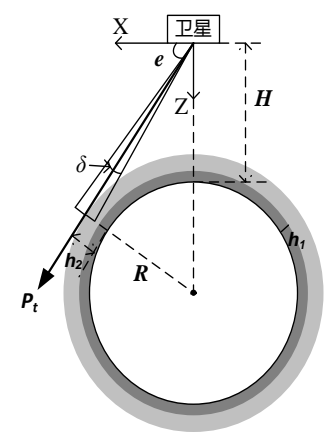

Figure 2 Background type related to sensor point schematic diagram

$N_{p h \mathrm{~d}}=\left\{\begin{array}{l}N_{1}, h>h_{2}(\text { Deep space }) \\ N_{2}, h_{1}<h<h_{2}(\text { Border background }) \\ N_{3}, h<h_{1}(\text { Earth's atmosphere })\end{array}\right.$

In the type(2): $N_{p h d}$ stands for the number of electrons of target and the background noise, The conversion formula from the background intensity to the number of electrons can be found in the literature[6].

(b) Thermal Noise

Thermal noise is formed by the random motion of electrons in the detector, as follows:

$\bar{n}_{\text {th }}^{2}=\frac{4 k T t}{q^{2} R_{0}}$

In the type(3): $T$ stands for the absolute temperature of sensor, $q$ is a charge amount, $R o$ is the effective load resistance.

(c) Readout Noise

Readout noise described all the electronic noise from the focal plane electronics to amplified current and to the A-D conversion[6], as follows:

$\bar{n}_{r o}^{2}=\frac{v_{r o}^{2} C_{\mathrm{int}}^{2}}{q^{2}}$

In the type(4): $V_{r o}$ stands for the detector readout noise voltage, $C_{i n t}$ is the detector equivalent integrating capacitor.

Therefore, we can draw the overall size of the system noise and the signal-to-noise is:

$\bar{n}=\sqrt{N_{p h \mathrm{~d}}+\bar{n}_{t h}^{2}+\bar{n}_{r o}^{2}}$

$S N R=\frac{N}{\bar{n}}$

Generally the tracking sensor maximum range is $6000 \mathrm{~km}$, we believe that the target can be detected if the radiation intensity of a point source can reach $30 \mathrm{~W} / \mathrm{sr}$, and noise equivalent flux density of space-based optical sensor is about $10^{-18} \mathrm{~W} / \mathrm{sr}$ magnitude, so we can obtain the minimum $S N R_{\min }$ value that the target can be detected is equal to about 8.3.

(d) Stray Light

After the external radiation source such as the moon, the sun and the light go though sensor lens hood to enter the detector, there formed the stray light. Especially when the viewing angle between scheduled sensor point and the moon, the sun is too small, stray light may cause the sensor to "blinding" or even burned. This is because the sunlight shoots directly into the view of the optical system will make tracking infrared sensor detector array is saturated, it is difficult to detect the target. To avoid this situation, the target detection by tracking sensor should meet the following conditions:

(1) Satellites is in earth umbra area, no sunlight can reach the satellite, this time the sun will not affect the target detection;

(2) satellite is not in earth umbra zone, daylight can reach the satellite, this time we have to 
ensure that the angle between the vector of satellite and sun and sensor point is bigger than the critical angle that the sunlight can influence tracking sensor detection .

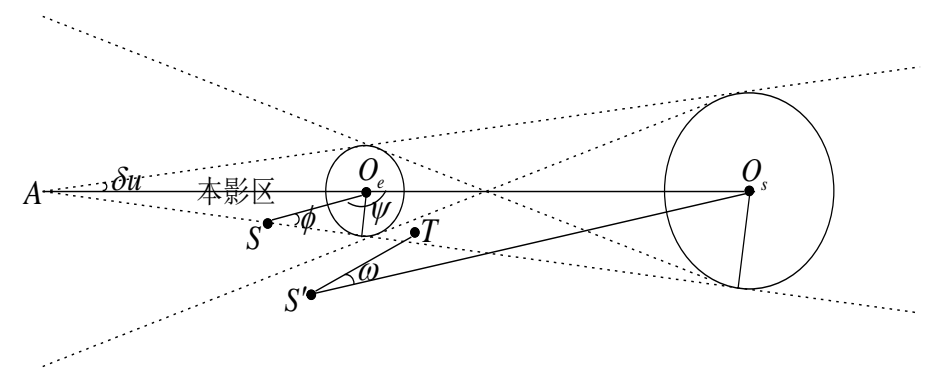

Figure 3 The impact of tracking sensors covered performance produced by sun

Shown in Figure 3, $O_{e}, O_{s}$ is geocentric and heliocentric, $S\left(S^{\prime}\right), T$ is satellite and target, the area all of sunlight shielded by the earth is umbra zone, $\delta u$ is umbra angle, $\psi$ is the angle between geocentric satellite vector and the Earth-Sun vectors, $\phi$ is the angle between geocentric satellite vector and umbra boundary. Assumed that $\psi_{\text {cri }}$ indicates the critical angle when satellite locates in the umbra zone boundary, then there is $\psi_{\text {cri }}=\pi-\phi+\delta u$, therefore stray light constraint is expressed as:

$$
\psi \geq \pi-\phi+\delta u \text { or }\left\{\begin{array}{l}
\psi<\pi-\phi+\delta u \\
\omega>\omega_{\text {cri }}
\end{array}\right.
$$

Further analysis, when the condition is $\omega>\omega_{\text {cri }}$, the specific suppression requirements is the irradiance of solar radiation to reach the surface of the detector in the operating waveband can not be higher than the sensor noise equivalent irradiance, According to the literature [7] we have an infrared sensor noise equivalent irradiance $E_{N E I}=2.17 \times 10^{-11} \mathrm{~W} / \mathrm{cm}^{2}$.

The definition of solar radiation stray light suppression ratio of sensor system is:

$$
R(\omega)=\frac{E_{d}(\omega)}{E_{0}}
$$

In the type(8), $E_{d}(w)$ stands for the irradiance in sensor focal plane when the angle between solar radiation and sensor optical axis is $\omega, E O$ is the irradiance of solar radiation in the operating waveband. For $3 \sim 5$ um working band, $E_{0}=2.17 \times 10^{-11} \mathrm{~W} / \mathrm{cm}^{2}$ And our system order for stray light suppression ratio is $10^{-8}$ magnitude, we can got the sun avoid angle, that is the critical angle, is $30^{\circ}$, which requires $\omega>30^{\circ}$.

Constraints are summarized as follows:

(1. sun, moon constraint angle must be greater than a critical angle, otherwise the camera will be damaged;

(2. SNR must be greater than the minimum value that can be detected, so we can ensure the re-capture probability is greater than $99.99 \%$, or we think the target is lost.

\section{The Constraints Condition Analysis In Target Stable Tracking Stage}

Targeting stable tracking stage refers to maintaining a high three-dimensional stable tracking accuracy after the reacquisition stage is successful, the process is the combined effects of the resource constraints, task constraints and time constraints, this article at this stage still adopts high tracking accuracy to optimize the function [3], but the main constraints aspect mainly take the sensor tracking performance and camera rotation angular velocity into consideration.

\section{(1) The Sensor Tracking Performance}

The effect or earnings that can be obtained from the sensor tracking missile process is called the sensor tracking performance, it is measured by tracking accuracy to. We can draw it though solving a two-dimensional image plane observation equation of two satellites to obtain the three-dimensional position, it is defined as follows [8]: 


$$
G D O P=\sqrt{\operatorname{Trace}(\boldsymbol{P})}
$$

\section{(2) The Camera Rotation Angular Velocity}

For optical warning systems in LEO, the position of the satellite and the target is changing in real time, so the camera rotation angular velocity is different with different geometry between satellite and target at different times, it includes pitch and azimuth angular velocity two parts and mainly influenced by the distance from the target to satellite. Because it has a significant impact on SNR at the re-capture stage, this constraint will be given out a separate analysis, simulation results are shown in Figure 4.

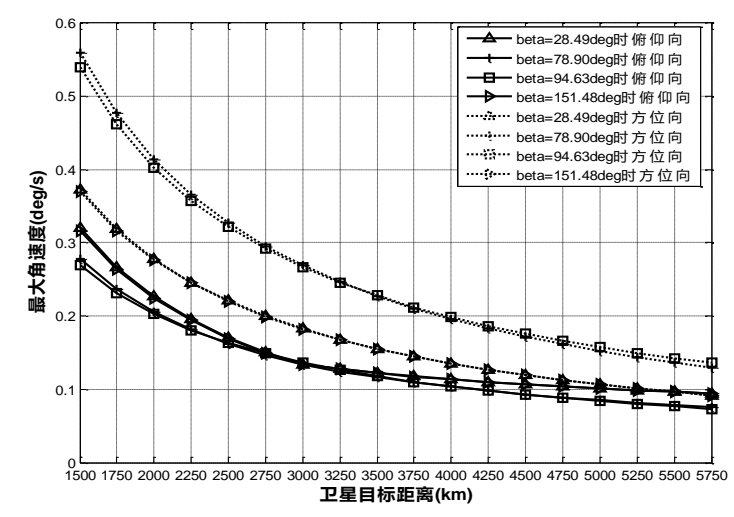

Figure 4 Effect of distance to camera rotation angle

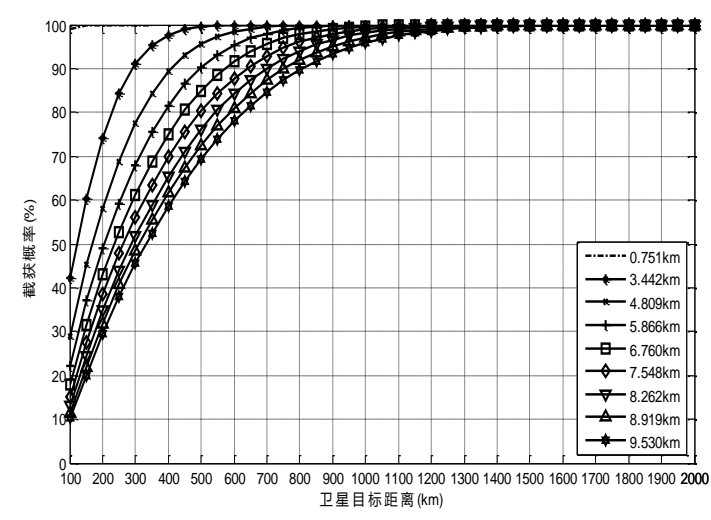

Figure 5 Effect of distance to Intercept Probability

As can be seen from the Figure 4, the rotation angular velocity decreases with increasing distance from the target to satellite, and therefore in the effective detection range of the tracking sensor, the farther from the target to satellite is, the smaller angular velocity is. And when the target is too close to the satellite, there is the problem the camera turned too fast. If the limited tracking sensor azimuth angular velocity is less than $0.5^{\circ} / \mathrm{s}$, then we can take the minimum effect distance as $2000 \mathrm{~km}$.

In addition, according to the impact on interception probability of the target satellite distance, the minimum distance $2000 \mathrm{~km}$ can be sure of more than $99.99 \%$ probability intercept. The related simulation results shown in Figure 5.

Constraints are summarized as follows:

(4. Satellite to target distance must be bigger than the minimum effective distance:2000km;

(4. select the tracking accuracy GDOP highest possible combinations of sensors.

\section{The Midcourse Optimization Model Including Recapture And Stable Tracking}

Combined with the analysis of tracking accuracy in stable tracking stage we have established the whole optimization functions as follows:

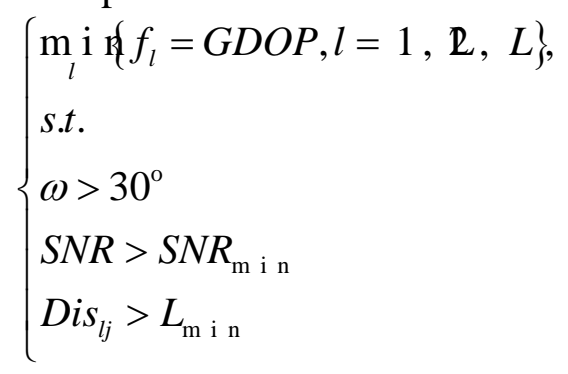

In the type(8), Dislj stands for the target to satellite distance, $l$ is sensor combination, the initial selection is based on a simple analysis of the target coverage [3], and here we are all in default a sensor combination including two sensors. 


\section{The Simulation Analysis}

\section{Emulation scene setting and performance evaluation parameters}

Constellation orbital parameters take 28/4/2/1596 / 77.8 [5] (the constellation represents a total of four orbital plane, seven satellites per orbital plane, orbit height $1596 \mathrm{~km}$, orbital inclination $77.8^{\circ}$, the phase factor is 2). Target parameters are shown in Table 1. Quantization step of predicting trajectory in the simulation take 50s, sensor observation interval is 1 second, the sight measurement error is $90 \mu \mathrm{rad}$. Simulation configured as Cuo2 E8400CPU, 4G memory desktops, and take a total of 50 times Monte Carlo experiment.

Do simulation verification on the midcourse sensor scheduling model the paper proposed, and compared with the literature [9]. Performance evaluation include: tracking position (velocity) error, target reacquisition loss rate, averaging time consuming.

Table 1 Target Parameters

\begin{tabular}{|c|l|l|c|c|}
\hline $\begin{array}{c}\text { Target } \\
\text { number }\end{array}$ & \multicolumn{1}{|c|}{$\begin{array}{c}\text { Emission point } \\
\text { target number }\end{array}$} & \multicolumn{1}{|c|}{ Fall points } & $\begin{array}{c}\text { Apogean } \\
\text { height }(\mathrm{km})\end{array}$ & Launch time (s) \\
\hline M1 & $E 24.000^{\circ}, N 80.000^{\circ}$ & $E 38.976^{\circ}, N 115.797^{\circ}$ & 1490.7 & 1 \\
\hline M2 & $E 35.000^{\circ}, N 137.000^{\circ}$ & $E 38.991^{\circ}, N 116.107^{\circ}$ & 1382.5 & 1 \\
\hline M3 & $E 23.000^{\circ}, N 120.000^{\circ}$ & $E 38.849^{\circ}, N 116.025^{\circ}$ & 1454.7 & 1 \\
\hline M4 & $E 65.000^{\circ}, N 144.000^{\circ}$ & $E 38.820^{\circ}, N 115.879^{\circ}$ & 1470.1 & 1 \\
\hline M5 & $E 19.000^{\circ}, N 138.000^{\circ}$ & $E 28.966^{\circ}, N 107.168^{\circ}$ & 1473.3 & 1 \\
\hline M6 & $E 19.000^{\circ}, N 138.100^{\circ}$ & $E 28.963^{\circ}, N 107.180^{\circ}$ & 1469.6 & 121 \\
\hline M7 & $E 19.000^{\circ}, N 138.000^{\circ}$ & $E 28.966^{\circ}, N 107.168^{\circ}$ & 1473.3 & 121 \\
\hline M8 & $E 19.000^{\circ}, N 138.100^{\circ}$ & $E 28.963^{\circ}, N 107.180^{\circ}$ & 1469.6 & 1 \\
\hline
\end{tabular}

Average calculation time: refers to under the above-described computer hardware environment, the time needed to complete each integral sensor scheduling algorithm execution

Target tracking error: refers to error estimate of the target position, velocity compared to its true value, it is measured by the root-mean-square error.

Target recapture loss rate: refers to the probability that the target is lost in the reacquisition.

\section{Test results}

Many midcourses tracking error statistics, calculate time-consuming, target reacquisition loss rate will show in Figure 6, Table 2 and Table 3.
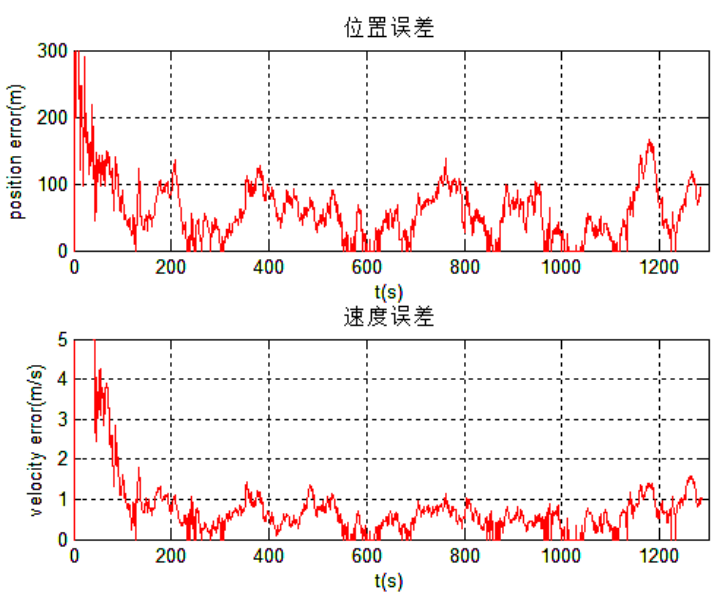

Figure 6 (a) position (velocity) error based on GDOP

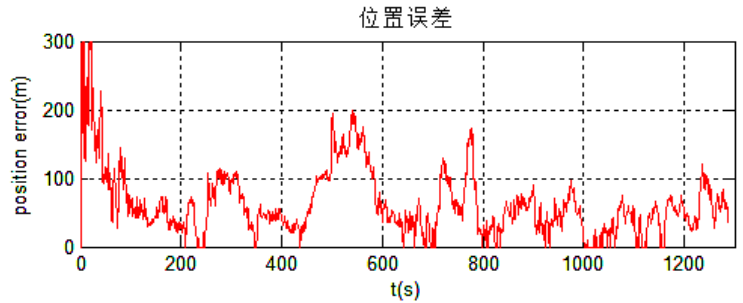

速度误差

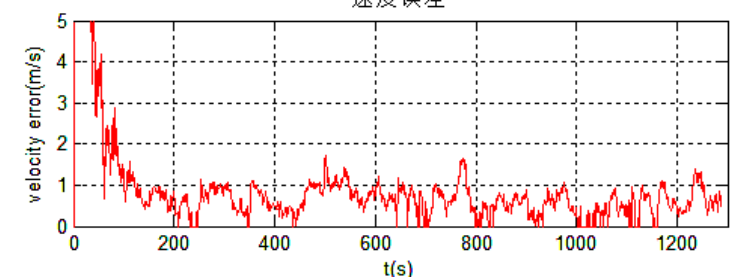

Figure 6 (B) position (velocity) error based on GDOP\&SNR 
Table 2 scheduling average calculation time-consuming comparison chart

\begin{tabular}{|c|c|c|}
\hline Optimal sensor function & GDOP & SNR+GDOP \\
\hline average calculation consuming time (s) & 22.37 & 32.11 \\
\hline
\end{tabular}

Table 3 The target loss rate of different scheduling function

\begin{tabular}{|c|c|c|c|c|c|c|c|c|c|}
\hline \multirow[b]{2}{*}{ scheduling function } & \multicolumn{8}{|c|}{ target loss rate $(\%)$} & \multirow{2}{*}{$\begin{array}{c}\text { Multi-target } \\
\text { total loss rate } \\
(\%)\end{array}$} \\
\hline & M1 & M2 & M3 & M4 & M5 & M6 & M7 & M8 & \\
\hline GDOP & 0 & 0 & 0 & 0 & 0.01 & 54.89 & 0 & 98.41 & 9.72 \\
\hline $\mathrm{SNR}+\mathrm{GDOP}$ & 0 & 0 & 0 & 0 & 0 & 0.01 & 0 & 0 & 0.001 \\
\hline
\end{tabular}

The analysis of Table 3: the initial allocation of sensor for M8 without signal to noise ratio restraint as follows:

Table 4 The initial allocation of sensor for M8

\begin{tabular}{|c|c|c|c|}
\hline & 1 & 2 & 3 \\
\hline 121 & 5 & 9 & \\
\hline 122 & 5 & 9 & \\
\hline 123 & 5 & 9 & \\
\hline 124 & 5 & 9 & \\
\hline 125 & 5 & 9 & \\
\hline 126 & 5 & 9 & \\
\hline 127 & 5 & 9 & \\
\hline 128 & 5 & 9 & \\
\hline 129 & 5 & 9 & \\
\hline 130 & 5 & 9 & \\
\hline
\end{tabular}

However, according to the satellite and target positional relationship, we calculated signal to noise ratio beyond the minimum SNR threshold, it will cause the loss of the target. The initial allocation sensor group changed after joining SNR constraint, Although its GDOP might not better than 5,9 sensor combination, but we got a higher probability of recapture and greatly reduce the target loss rate.

\section{Conclusion}

In this paper, based on previous studies on sensor scheduling model measured by GDOP, we take SNR into a real important consideration in recapture process to present the new scheduling model more combined with LEO space-based sensor satellites network and target actual motion. As can be seen from the above simulation results, although the position (velocity) error of the new sensor scheduling optimization model was slightly increased, the scheduling time-consuming calculation was decreased, the target loss rate has improved significantly, it has more advantages from full-warning constellation performance standpoint. In summary, GDOP optimization function model which added SNR constraint can be closer to the actual warning situation, it makes constellations achieve better overall performance. In addition, the increase of the target loss rate has a strong selectivity on the scene, universality of this model needs further study.

\section{References}

[1] Lihua $\mathrm{Wu}$, The research on orientation estimation of ballistic missile boost phase based on a single satellite in geosynchronous orbit [master's paper], National University of Defense Technology.

[2] Samuel Blackman,Robert Popoli. Design and Analysis of Modern Tracking Systems[M]. BostonLondon: Artech 
House, 1999: 967-1065.

[3] Wang Bo,Yiyu Zhou. The research on sensor resource scheduling algorithm in LEO Infrared Early Warning Constellation ,master and doctor full-text database 2010

[4] Wang Bo,Yiyu Zhou,Xu Dan,An Wei. The real-time sensors scheduling model in LEO constellation[J]. Journal of Basic Science and Engineering,2011

[5] Michael J. Cantella.Theinfred\&electro-optical systems handbook [J].Washington USA:SPIE Press.1993,159-205

[6]Weizhen Chen,Chunhua Zhang,Xiaodong zhou,The study on Photometric properties of space target and imaging SNR [J]. Infrared Technology,2007,29 (12): 716-719

[7] Jinxing Niu,Renkui Zhou,Zhaohui Liua and so on. Stray light analysis and calculation of space-based infrared detection system [J]. :Acta Optica Sinica,2010,30 (3): 768-771

[8] Wang Bo,Yiyu Zhou,Jianhua Lu,An Wei.Research on sensor management algorithm of STSS based on real-number particle swarm optimization[J].Journal of System Simulation, 25(7): $1135-1140$

\section{About the Author}

Yuanyuan Yang: Female, born in 1992, College of Electronic Science and Engineering, National University of Defense Technology Graduate, main research directions: multi-sensor management scheduling.

contact address:

Changsha, capital of Hunan Province, College of Electronic Science and Engineering, National University of Defense Technology, space-based warning room

Yang Yuanyuan (received)

Zip: 410073, China;

Phone: 18373145477; Email: 352906956@qq.com 